\title{
Robust and Fast UV-HPLC Method for Biotransformation Analysis of Azecines
}

\author{
Stephanie Zergiebel ${ }^{1} \cdot$ Andreas Seeling $^{1}$ (I)
}

Received: 29 September 2020 / Revised: 10 December 2020 / Accepted: 3 January 2021 / Published online: 23 January 2021

(c) The Author(s) 2021

\begin{abstract}
Dibenzoazecines are a new class of drug candidates for the treatment of schizophrenia. Compared to the drugs currently used in therapy, the azecines have a novel mechanism of action. Thus, they have the potential to cause fewer side effects compared to the standard therapy with a constant high neuroleptic potency. This theory was substantiated by comparative in vivo tests with haloperidol and risperidone. Seventeen new azecine derivatives have already been tested furthermore of stability, physicochemical parameters, pharmacokinetics including esterase cleavage, stability in simulated gastrointestinal fluid, stability at different $\mathrm{pH}$ values and determination of octanol/water-partition coefficients. For these substances, class is still a lack of information concerning the metabolism. Therefore, the present study investigated and developed a reliable and reproducible gradient reversed-phase HPLC-UV method to determinate the lead structure LE404 alongside emerging metabolites in compliance with international requirements like ICH guidelines and the European Pharmacopoeia. Up to now, there is no innovative method suitable for such a determination. Chromatographic separations were achieved with a phenomenex ${ }^{\mathrm{TM}}$ Gemini column $(5 \mu \mathrm{m} \mathrm{C} 18110 \AA$, $250 \times 4.60 \mathrm{~mm})$ using a mixture of acetonitrile/potassium dihydrogen phosphate buffer ( $4 \mathrm{mmol} \mathrm{L}^{-1}, \mathrm{pH} 2.5$ ) as mobile phase. The gradient method flow rate was $1.0 \mathrm{~mL} \mathrm{~min}^{-1}$, and UV detection was made at $220 \mathrm{~nm}$. The optimized HPLC method was found to be specific, accurate, reproducible and robust for determination of LE404.
\end{abstract}

Keywords Azecines $\cdot$ HPLC-UV method development $\cdot$ Metabolism $\cdot$ Neuroleptics

\section{Introduction}

Schizophrenia occurs in $1 \%$ of the general population [1] and is a disease that belongs to the endogenous psychoses, which is treated with neuroleptics medication [2]. Although there has been an almost linear increase in the prescription of atypical neuroleptics since 2008 [3], the overall antipsychotic efficacy of neuroleptics is described as minimal compared to placebo [4]. The lack of compliance due to side effects also limits therapeutic success [5]. This shows that there is an ever-increasing need for neuroleptics, but currently available drugs cannot provide a symptom-free life for the most of the schizophrenia diseased.

Dibenzoazecines represent a new class of potential neuroleptics with high-affinity dopamine (D) and serotonin

Andreas Seeling

andreas.seeling@uni-jena.de

1 Institute of Pharmacy, Friedrich-Schiller University Jena, Philosophenweg 14, 07743 Jena, Germany
$\left(5-\mathrm{HT}_{2 \mathrm{~A}}\right)$ receptor antagonism $[6,7]$. Thus, azecines show a novel mechanism of action with preferential binding to the D1 receptor subtype $\left(D_{1}, D_{5}\right)$ [8]. Moreover, tests have shown that they bind at the 5- $\mathrm{HT}_{2 \mathrm{~A}}$ receptor [9]. The antagonistic activity on the mentioned receptors was evidenced by functional calcium assays $[8,9]$. In in vivo tests, the neuroleptic potency of 7-methyl-5,6,7,8,9,14-hexahydrodibenzo[d,g] azecin-3-ol (LE404) (Fig. 1), a promising representative of the substance class, was demonstrated [9]. In addition, the fivefold higher therapeutic range of LE404, compared to the standard substances haloperidol and risperidone, characterizes the azecines as potentially useful drugs against schizophrenia [9]. In further research, LE404 prodrugs with altered pharmacokinetic properties were synthesized $[10,11]$ and screened in in vivo and in vitro assays [12]. However, there is no information about the metabolism of azecines at this time. Therefore, the aim of this study is the development of a rapid, simple, sensitive, reproducible and cost-effective HPLC-UV method for the identification and quantification of the LE404 lead structure alongside emerging metabolites 


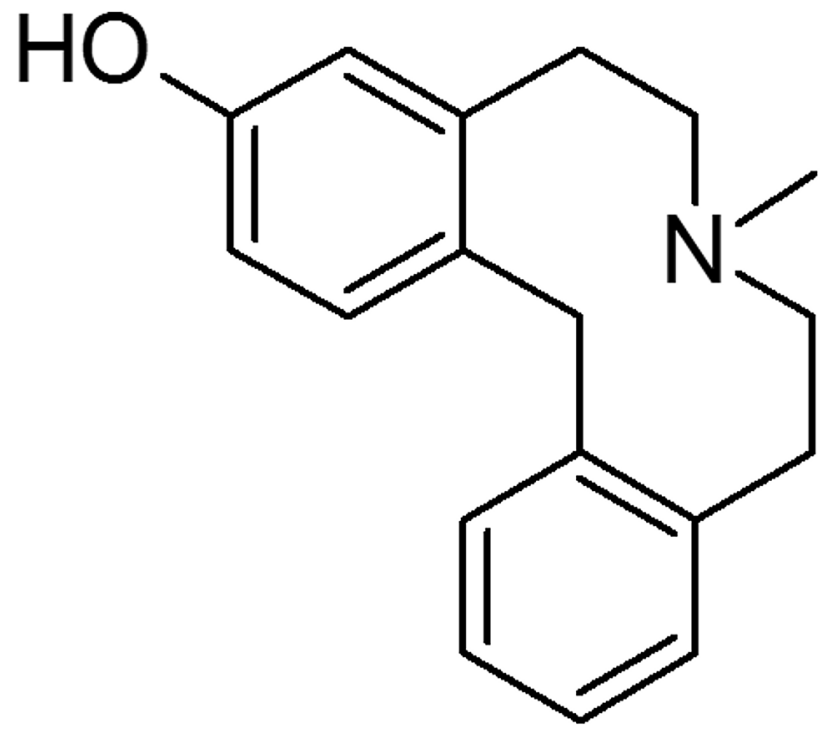

Fig. 1 Lead structure 7-methyl-5,6,7,8,9,14-hexahydrodibenzo[d,g] azecin-3-ol (LE404)

(e.g., produced by reaction with human liver microsomes). So far, no HPLC methods are known to handle this problem. The intention is to develop an analytical method which, on the one hand, enables future metabolites to be isolated and, on the other hand, provides a basis for stability and stress tests. In addition, a full validation should be carried out to show compliance with international requirements for analytical methods for the quality control of pharmaceuticals (ICH guidelines [13] and European Pharmacopoeia [14]). The validation parameters included linearity, sensitivity, accuracy, precision and robustness. Furthermore, a system suitability test was carried out.

\section{Materials and Method}

\section{General}

All solvents and reagents were purchased from Merck (Germany), Sigma-Aldrich Chemie Ltd. (Germany), VWR International Ltd. (Germany) or Fisher Scientific Ltd. (Germany) and correspond to the purity "p.a.". For preparation of mobile phases, HPLC gradient grade acetonitrile (HiPerSolv CHROMANORM ${ }^{\mathrm{TM}}$, VWR International, Belgium) and deionized water (TKA GenPure water system, Thermo Electron LED Ltd., Germany) were used. The tested lead structure LE404, synthesized in the Institute of Pharmacy Jena $[11,15]$, is shown in Fig. 1. The phosphate buffer $\mathrm{pH} 7.4$ used for the metabolism test contains $26.5 \mathrm{~g} \mathrm{Na}_{2} \mathrm{HPO}_{4} \cdot 12$ $\mathrm{H}_{2} \mathrm{O}$ and $2.3 \mathrm{~g} \mathrm{KH}_{2} \mathrm{PO}_{4}$ dissolved at $1000 \mathrm{~mL}$ with deionized water. The $\mathrm{pH}$ was adjusted to 7.4 with $10 \%(\mathrm{~m} / \mathrm{v}) \mathrm{NaOH}$ or $10 \% \mathrm{H}_{3} \mathrm{PO}_{4}(\mathrm{~m} / \mathrm{v})$. For the study of liver metabolism,
CellzDirect ${ }^{\mathrm{TM}}$ Human Liver Microsomes (18-39, 25 Donor Pool, volume $=0.5 \mathrm{~mL}$, Catalog \# HMMC-18-PL036, Thermo Fisher Scientific) were used.

\section{Instrument and Chromatographic Conditions}

The metabolism assay of the azecine derivative was monitored by HPLC analysis. The decrease in the amount of the starting material was determined by using $\beta$-naphthol as an internal standard. HPLC analyses were performed using a LC-10 AS pump, an auto-injector SIL-10 A with an UV detector SPD-10A in combination with a communication bus module SCL-10AVP. Integration and calculation were done with Class LC10 $0^{\mathrm{TM}}$ software (all parts Shimadzu Europe). Mobile phase A contains $4 \mathrm{mmol} \mathrm{L}^{-1}$ potassium dihydrogen phosphate-buffer $\mathrm{pH} 2.5$ and mobile phase $\mathrm{B}$ acetonitrile. A membrane filter of $0.2 \mu \mathrm{m}$ porosity was used to filter the mobile phase (RC-Membranfilter, Sartorius, Germany), and then, the mobile phase was degassed by sonification. The gradient elution was as follows: $0-17.0 \mathrm{~min} 90-65 \%$ A, 17.0-25.0 min 65-20\% A, 25.0-26.0 min 20-90\% A, 26.0-36.0 min $90 \%$ A. Separations were achieved using a C18 reversed-phase column (phenomenex ${ }^{\mathrm{TM}}$ Gemini $5 \mu \mathrm{m}$ C18 $110 \AA$ A, 250×4.60 mm, Phenomenex Inc., Germany) and a precolumn (phenomenex ${ }^{\mathrm{TM}}$ Security Guard Cartridge Gemini C18, 4×3 mm, Phenomenex Inc., Germany). To study the robustness of the method, the separation behavior on a C8 reversed-phase column (LiChroCART ${ }^{\mathrm{TM}}$ 250-4 HPLC-Cartridge, Superspher ${ }^{\mathrm{TM}} 60$ RP-8, Merck, Germany) with a precolumn (LiChrospher ${ }^{\mathrm{TM}} 100$ RP-8 endcapped, $5 \mu \mathrm{m}$, Merck, Germany) was investigated under otherwise identical experimental conditions. Chromatography was conducted at ambient temperature at a flow rate of $1 \mathrm{~mL} \mathrm{~min}{ }^{-1}$, and the detection wave length was $220 \mathrm{~nm}$. The volume of the sample solution injected was $50 \mu \mathrm{L}$. Thiourea was used to determine the dead time.

\section{Sample Preparation}

The sample preparation and the incubation were carried out on the basis of the literature [16]. Commercial human liver microsomes (fractionated to $5 \mu \mathrm{L}$ microsomes suspension per vial directly after delivery and stored at $-80^{\circ} \mathrm{C}$ ) were rapidly thawed at $37^{\circ} \mathrm{C}$, suspended in $183 \mu \mathrm{L}$ pre-tempered $\left(37^{\circ} \mathrm{C}\right)$ phosphate buffer $\mathrm{pH} 7.4$ and left for the duration of the experiment in a water bath at $37{ }^{\circ} \mathrm{C}$ with gently panned throughout to ensure a homogeneous distribution of active ingredients and adequate oxygenation. In each vial, $2 \mu \mathrm{L}$ of the test substance $\left(1 \mathrm{~mol} \mathrm{~L}^{-1}\right.$ LE404 in acetonitrile) was introduced (final organic solvent concentration $=1 \%$ ) and preincubated for $5 \mathrm{~min}$. Then, the reaction was started by addition of $10 \mu \mathrm{L}$ freshly prepared NADPH solution (0.02 mol L ${ }^{-1} \mathrm{NADPH} \cdot \mathrm{Na}_{4}$ in phosphate buffer $\mathrm{pH}$ 7.4). 
After the incubation period of $60 \mathrm{~min}, 300 \mu \mathrm{L}$ acetonitrile was added to each sample vial and vortexed to terminate the reaction and for complete denaturation. For phase separation, $15 \mathrm{mg}$ of magnesium chloride per sample vial was added. The samples were centrifuged (4000 rpm, $5 \mathrm{~min}$ ), placed in an ice bath and after phase separation, and $100 \mu \mathrm{L}$ of the organic phase was transferred to HPLC vials, mixed with $50 \mu \mathrm{L}$ of distilled water and were analyzed. Sample preparation and incubation were carried out under exclusion of light.

\section{Analytical Validation}

\section{Linearity and Range}

The limit of detection (LOD) and limit of quantification (LOQ) for LE404 were determined based on the standard deviation of the response and the slope according to the ICH Guideline Q2 (R1) [13] by injecting a series of diluted solutions of LE404 (8 concentrations between $5 \times 10^{-3} \mathrm{~mol} \mathrm{~L}^{-1}$ and $10^{-6} \mathrm{~mol} \mathrm{~L}^{-1}, 3$ times per concentration) to get a calibration curve. Standard deviation, slope, intercept and coefficient of determination $\left(R^{2}\right)$ of the calibration curve were calculated to ascertain linearity of the method. For the formed metabolites, the LOD and LOQ of the method were evaluated considering the analyte concentration via the signalto-noise ratio $(\mathrm{S} / \mathrm{N})$ because no reference substances for the metabolites are available. The LOD represents the analyte concentration that would yield a $\mathrm{S} / \mathrm{N}$ value of 3 , and the LOQ represents the analyte concentration that would yield a $\mathrm{S} / \mathrm{N}$ value of 10 [13].

\section{Accuracy}

With the method developed, three concentrations of LE404 $\left(4.5 \times 10^{-5} \mathrm{~mol} \mathrm{~L}^{-1}, 7.5 \times 10^{-4} \mathrm{~mol} \mathrm{~L}^{-1}, 3.5 \times 10^{-3} \mathrm{~mol} \mathrm{~L}^{-1}\right)$ that were not part of the calibration were measured three times each to check the accuracy of the method. Using the regression equation, the concentrations could be determined by calculation.

\section{System Suitability}

The resolution $\left(R_{S}\right)$, selectivity $(\alpha)$, peak symmetry $\left(A_{\mathrm{S}}\right)$, theoretical plate number $(N)$ and theoretical plate height $(H)$ were determined according to the European Pharmacopoeia 9.0 [14].

\section{Precision}

In addition, the intra-day precision and the inter-day precision of the method were determined by injections of a standard solution containing LE404 and $\beta$-naphthol as internal standard. Six injections were performed each day for three consecutive days. The inter-day precision was calculated by determining the retention times on 18 different days. The intra-day precision and the inter-day precision for the substances which are supposed to be metabolites were also determined.

\section{Results and Discussion}

\section{HPLC Method Development}

Because the metabolites are so far unknown, no estimation of their quantity and polarity could be made before the method development. It can be assumed that the majority of the metabolites will be more hydrophilic than the starting substance LE404 [17]. Therefore, the HPLC method was started with a high aqueous content of $90 \%$ and lowered the proportion of the aqueous phase to $65 \%$, within the first 17 min, with a flat linear gradient. The second linear gradient is steeper and increases the solvent content to $80 \%$ after a further $8 \mathrm{~min}$. This concept is intended to ensure that even occurring lipophilic metabolites can be separated and detected. LE404 elutes in the last quarter of the first gradient, with an average of $13.43 \mathrm{~min}(n=8)$. At the end, an equilibration phase of 10 min takes place to restore the starting conditions for the next run. The method has been optimized so that an analysis and equilibration time of $30 \mathrm{~min}$ are not exceeded. Figure 2a shows the separation of the LE404 and the supposed metabolites with the C18 reversed-phase column after incubation. The reference chromatogram Fig. $2 b$ illustrated the treatment of LE404 under incubation conditions with NAPDH but without microsome supplementation. However, the three emerging impurities also occur in the same amount in the extraction of LE404 under the given conditions $\left(37^{\circ} \mathrm{C}, 65 \mathrm{~min}\right.$.) without addition of NADPH and microsomes. It can be assumed that these impurities occur over the long-time storage and not because of the influence of cofactors, microsomes and the increased temperature $\left(37^{\circ} \mathrm{C}\right)$ or the solvent. The purity of the used LE404 was over $98.26 \%$ at the time of the microsomes tests, despite of the three impurities.

During the microsomes tests, it was found that three impurities occurred in the used test substance (LE404); therefore, the stability of LE404 was examined over a period of 21 days. Solid LE404 was stored at $25 \pm 2{ }^{\circ} \mathrm{C}$ in daylight and examined by the HPLC method used for metabolism. The resulting chromatograms are shown in Fig. 3. The peak area of LE404 decreases by $37.23 \%$ over this period.

In order to investigate the nature, extent and prerequisites for this degradation, a semi-preparative separation method was necessary. Because of the same retention times and the increasing peak areas over the incubation with the microsomes, it can 


\section{LE404}

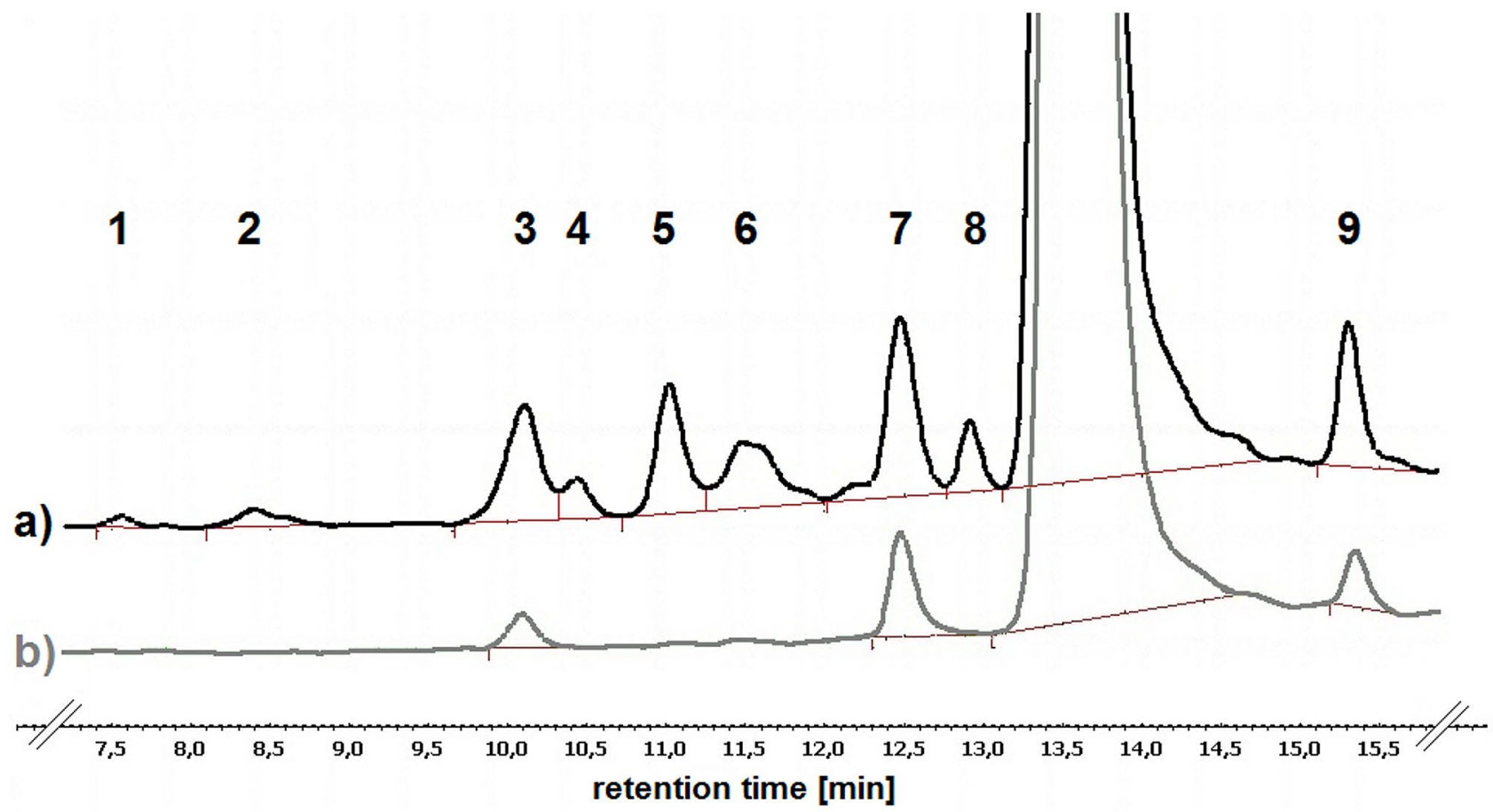

Fig. 2 HPLC-UV chromatograms (C18 reversed-phase column) showing a separation of LE404 and its metabolites (peak 1-9) after incubation with NADPH and microsomes and $\mathbf{b}$ separation of LE404 and its three degradation products (peak 3, 7, 9) after incubation with NADPH

be speculated that these three degradation products also arise metabolically. Furthermore, six additional potential metabolites could be detected (Fig. 2). According to Fig. 2b, it can be excluded that these substances occur because of the influence of cofactors, microsomes or the increased temperature $\left(37^{\circ} \mathrm{C}\right)$. Moreover, the suitability of the developed method for an $\mathrm{C} 8$ reversed-phase column was investigated with a LE404 sample containing the three defined breakdown products, because no reference substances are present for the multiplicity of peaks resulting after the incubation. Table 1 compares the resulting retention times and peak symmetries.

The peak symmetry is worse for all substances except $\beta$-naphthol when using the $\mathrm{C} 8$ reversed-phase column. Peak A and Peak B elute at an identical time and so they are not separable by this method. From a concentration of $5 \times 10^{-4} \mathrm{~mol} \mathrm{~L}^{-1}$, LE404 is no longer baseline separated from $\beta$-naphthol. The method developed for a $\mathrm{C} 18$ reversedphase column is thus not usable for a $\mathrm{C} 8$ reversed-phase column.

\section{Analytical Validation}

\section{Calibration of LE404}

The calibration curve for LE404 was found to be linear in the range of $5 \times 10^{-3} \mathrm{~mol} \mathrm{~L}^{-1}$ to $10^{-6} \mathrm{~mol} \mathrm{~L}^{-1}$. The calculated
LOD was $6.71 \times 10^{-7} \mathrm{~mol} \mathrm{~L}^{-1}$, and the calculated LOQ was $2.03 \times 10^{-6} \mathrm{~mol} \mathrm{~L}^{-1}$ with a coefficient of determination of 0.9991 . The deviation of the true values of three concentrations (that were not part of the calibration) from the measured value was below $2 \%$. So it suggested good accuracy and an excellent linearity of this method. The precision of the chromatographic system was tested by intra- and inter-day reproducibility of retention times and peak area (Table 2 ). The low values of intra- and inter-day RSD values for the retention times and also for the peak areas indicate the high precision of the chromatographic system.

\section{System Suitability for the Separation of the LE404 Metabolites}

To test the suitability of the HPLC method for the study of LE404 liver metabolism, a variety of system tests were performed. The examination of the intra- and inter-day reproducibility provides information about the precision of the system (Table 3). The RSD values are higher than those of the separate injection of LE404. This may be due to extracted matrix constituents which cause interactions with the column and the analytes. These matrix components cannot be standardized and are available in different quality and quantity for each donor, depending on the living conditions. Nevertheless, the somewhat higher RSD values of the 
Fig. 3 Stability test of LE404 (0-21 d, 25 $\pm 2{ }^{\circ} \mathrm{C}$, daylight), HPLCUV chromatograms (C18 reversed-phase column) showing a $0 \mathrm{~d}$, LE404 pure; b 7 d, LE404 and impurity 9; c 14 d, LE404 and impurity 9; 21 d, LE404 and impurity 3,7 and 9

retention times show that a clear assignment of the peaks over their retention times is possible, and thus, the reproducibility of this method has been demonstrated. The repeatability (intra-day assay) ranges between 1.15 and $25.81 \%$ and the reproducibility (inter-day assay) ranges between 2.16 and $24.65 \%$ based on the deviation of the peak height. This large variation is due to the comparatively large deviations in the peak height of peaks 6 and 8 (Fig. 2). Peak 6 and peak 8 showed optically no typical Gaussian peak shape. Thus, these two peaks could consist of two or more substances. This mixture results in a low reproducible peak shape and peak height. After separating the metabolites with the method presented here, a further analytical method would have to be developed for peak 6 and peak 8 in order to separate the substances contained in these peaks and make them accessible for characterization. In view of the comparatively small peak areas of peak 6 and 8 , which are further reduced by the number of substances contained per peak, identification is initially not in the foreground. The main metabolites (peaks 3, 7 and 9) are in the focus for future investigations.

Further data that demonstrate the suitability of the experimentally determined HPLC method are summarized in Table 4.

$L O D / L O Q$ : The LOD and LOQ for the metabolites were evaluated considering the analyte concentration that would yield a $\mathrm{S} / \mathrm{N}$ value of 3, respectively, a $\mathrm{S} / \mathrm{N}$ value of 10 . With the sample preparation mentioned here, all metabolites except peaks 1 and 2 fulfill the specification according to ICH guideline [13]. Peaks 4, 6 and 8 are detectable by this method but not quantifiable. These results indicate that the proposed HPLC method is sufficiently sensitive for the determination of LE404 metabolites and limited applicable for quantification.

Peak resolution $\left(R_{S}\right)$ : As another validation parameter, the peak resolution $\left(R_{S}\right)$ was determined. All peaks show baseline separation except for peaks 3 and 4 as well as for peaks 5 and 6 . The integration of two not baseline separated Gaussian peaks is nevertheless possible with a perpendicular and by triangulation [18] or by deconvolution [19]. Thus, the resolution of the metabolites is sufficient for quantification.

Peak symmetry $\left(A_{S}\right)$ : The aim of a chromatographic separation is symmetrical Gaussian peaks. According to the European Pharmacopoeia [14], an $A_{\mathrm{S}}$ value of 1 indicates symmetry. The calculated peak symmetry $\left(A_{S}\right)$, presented in Table 4, demonstrates that peaks 1 and 3 show a slight

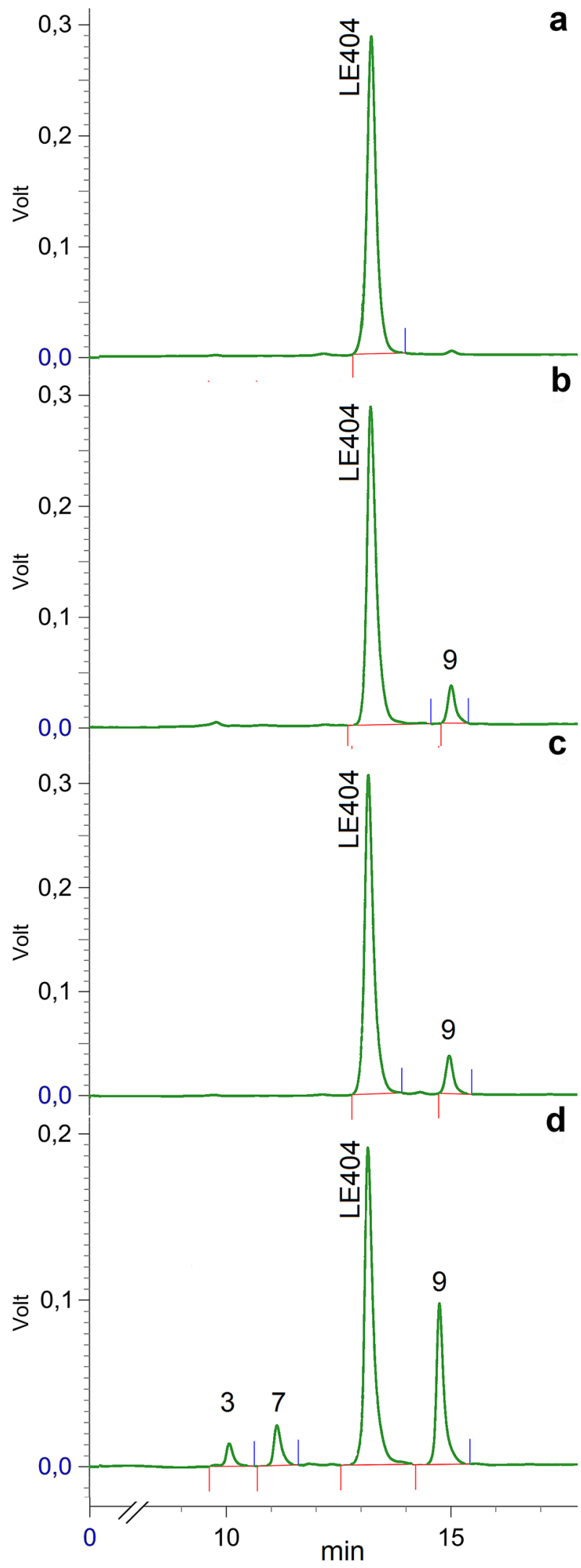


Table 1 Comparison of retention times $\left(\mathrm{t}_{\mathrm{R}}\right)$ and peak symmetries $\left(A_{S}\right)$ for C8 reversed-phase and $\mathrm{C} 18$ reversed-phase column

\begin{tabular}{llllllll}
\hline Column & \multicolumn{2}{l}{ C8 reversed phase $(n=8$, mean $)$} & & \multicolumn{3}{l}{ C18 reversed phase $(n=8$, mean $)$} \\
\cline { 2 - 3 } & $t_{\mathrm{R}}(\min )$ & $\mathrm{RSD}(\%)$ & $A_{\mathrm{S}}$ & & $t_{\mathrm{R}}(\min )$ & $\mathrm{RSD}(\%)$ & $A_{\mathrm{S}}$ \\
\hline Peak 3 & 16.83 & 0.37 & 1.90 & & 10.19 & 0.65 & 0.93 \\
Peak 7 & & & & 12.54 & 0.42 & 1.15 \\
LE404 & 22.54 & 0.63 & 1.75 & & 13.43 & 0.29 & 1.47 \\
Peak 9 & 24.03 & 0.29 & 1.43 & & 15.28 & 0.38 & 1.16 \\
$\beta$-Naphthol & 21.82 & 0.21 & 1.00 & & 23.68 & 0.03 & 1.16 \\
\hline
\end{tabular}

fronting. The other peaks show a slight tailing. The European Pharmacopoeia [14] requires that for quantification the symmetry factor of the peaks must be between 0.8 and 1.5. This is fulfilled by the present method. In spite of the fact that the calculated peak symmetry is within the specifications, Fig. 2 shows that peaks 6 and 8 are not Gaussian peaks. As already discussed, these two peaks probably consist of 2-3 substances, which cannot be sufficiently separated with the method developed here.

Selectivity $(\alpha)$ : The selectivity $(\alpha)$ a is also a criterion of the quality of an analytical separation of substances. According to the European Pharmacopoeia, it should be greater than 1 by definition [14]. All peaks correspond to this specification.

Theoretical plate number $(N)$ : Furthermore, the theoretical plate number $(N)$ is given in Table 4 for each substance and the resulting theoretical plate height $(H)$.

\section{Transfer from analytical to semi-preparative separation}

A sufficient stability over the duration of storage to the application of a drug is of great importance. On the one hand, the concentration of the drug should not decrease significantly; on the other hand, it must be clear which degradation products are formed and whether they may even be toxic. Drugs in preparations should generally be storable for 3-5 years. Storage stabilities below 2 years are unacceptable in most cases [20]. During the investigations, it was shown that three degradation products occurred in the LE404 samples, which increased in concentration over the period of storage (Fig. 3). These impurities were very easy to separate with the HPLC method shown here. For this reason, the HPLC method presented here is the starting point for future studies of the stability of LE404 according to ICH Guideline Topic Q 1 A (R2)/Stability Testing of new Drug Substances and Products [21], which are essential for a new drug candidate. In order to be able to identify these degradation products in the future, a transfer of the analytical separation into a semi-preparative separation was strive for. The peak resolution was for (peak 3)-(peak 7) 8.78, (peak 7)-(LE404) 2.93 and (LE404)-(peak 9) 5.55. Due to the excellent resolution between the LE404 peak and the degradation products, the use of this method is also possible for a semi-preparative separation, because even with a slight overcharge of the column, the peaks will probably still be baseline separated. In addition, it can be assumed that the area of LE404 decreases significantly over time under the conditions of the future stability tests according to ICH Guideline Topic Q 1 A (R2) [21] while the other areas increase. This also promotes a consistently good separation even in semi-preparative conditions. The phosphate buffer of the mobile phase used here can be removed by extraction after the semi-preparative separation, because LE404, with a octanol/water-partition coefficient $\left(\log \mathrm{P}_{\mathrm{O} / \mathrm{W}}\right.$ ) of 3.33 [12], can be extracted very good by organic solvents. Thus, the use of buffer substances in the mobile phase is not an exclusion criterion for a semipreparative separation.

\section{Conclusion}

The azecines are a promising new class of drugs for the potential treatment of schizophrenia. After the in vivo testing for efficacy and side effects $[9,10]$, the next major research area is the metabolism of this class of compounds. Until now, there has been no HPLC method for the separation and detection of azecines and their metabolites. In this study, a HPLC-UV method for the qualitative and quantitative analysis of the lead structure LE404 and its metabolites was developed. Due to the high structural homology of azecines, it is very likely possible to adapt this method to other derivatives as well. In addition, LE404 occurs as postulated intermediate at most of the azecines [12]. The proposed method requires analytical devices that are standard equipment in most laboratories, involves a low effort in sample preparation and allows the analytical procedure to be performed within a relatively short time. This method permits the unequivocal identification of LE404 in addition to its metabolites. Because the calibration was done over a very wide concentration range with an excellent regression coefficient, it is possible to use the method for a variety of metabolism studies in different concentration ranges. The validation procedure, according to ICH-guidelines [13] and the European Pharmacopoeia [14], confirmed that this HPLC method affords reliable analysis of these substances 

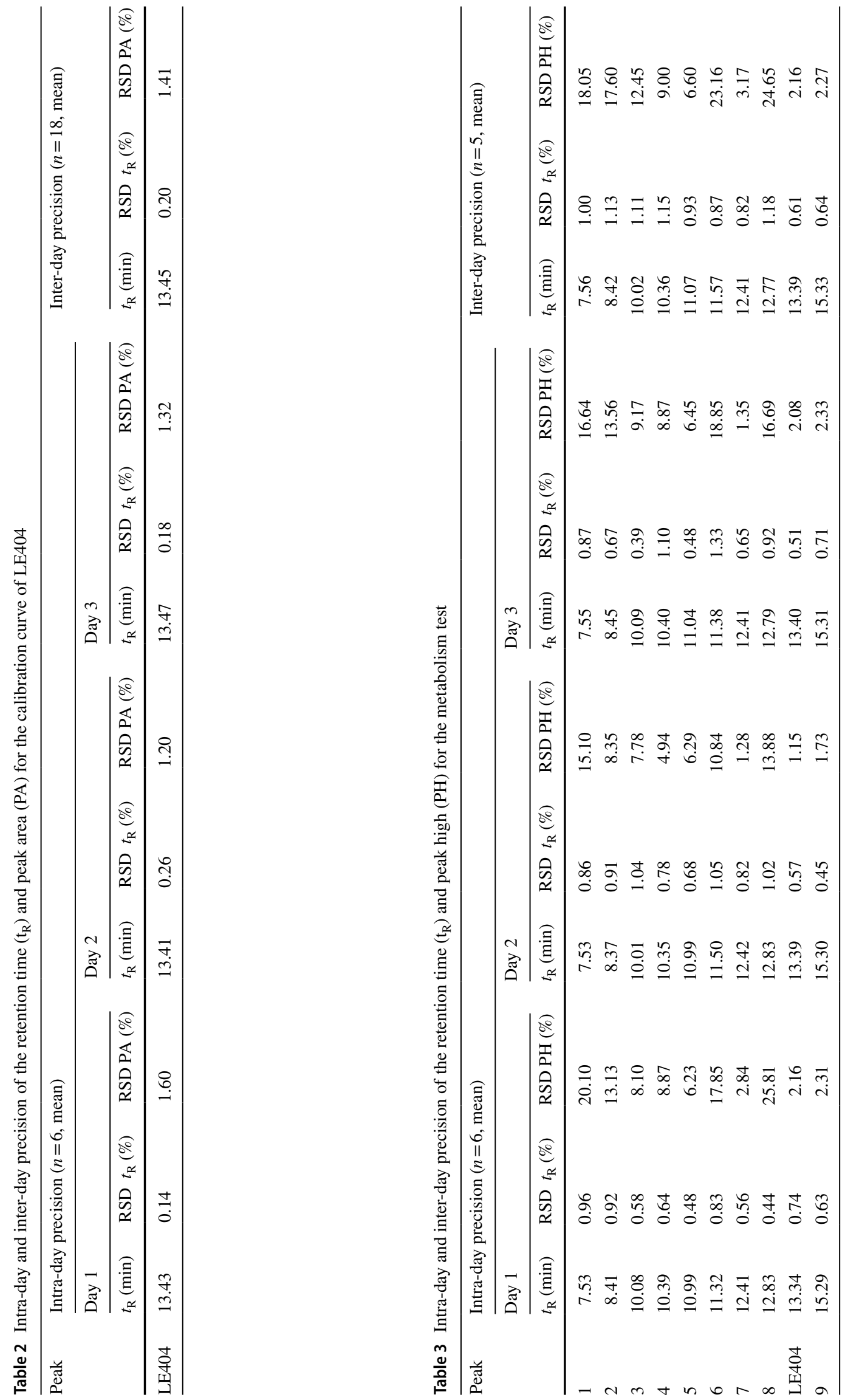
Table 4 System suitability report for the separation of LE404 and its metabolites $(x=S / N$-ratio fulfill not the specification according to $\mathrm{ICH}$ guideline [13], $\checkmark=\mathrm{S} / \mathrm{N}$ ratio fulfill the specification according to $\mathrm{ICH}$ guideline [13], peak symmetry $\left(A_{\mathrm{S}}\right)$, theoretical plate number $(N)$, theoretical plate height $(\mathrm{H})$ signal-to-noise ratio $(\mathrm{S} / \mathrm{N})$, limit of detection (LOD), limit of quantification (LOQ), selectivity $(\alpha)$, peak resolution $\left(R_{S}\right)$ )

\begin{tabular}{lllrlllll}
\hline Peak & \multicolumn{1}{l}{$A_{\mathrm{S}}$} & $N$ & $\mathrm{H}(\mu \mathrm{m})$ & $\mathrm{S} / \mathrm{N}$ & $\mathrm{LOD} / \mathrm{N}>3$ & $\begin{array}{l}\text { LOQ } \\
\mathrm{S} / \mathrm{N}>10\end{array}$ & $\alpha$ & $R_{S}$ \\
\hline 1 & & & & & & & \\
2 & 0.87 & 14,065 & 17.77 & 0.98 & $\times$ & $\times$ & - & - \\
3 & 1.35 & 6254 & 39.97 & 2.35 & $\times$ & $\times$ & 1.19 & 2.48 \\
4 & 0.93 & 9056 & 27.60 & 11.18 & $\checkmark$ & $\checkmark$ & 1.32 & 4.03 \\
5 & 1.11 & 11,419 & 21.89 & 3.92 & $\checkmark$ & $\times$ & 1.05 & 0.82 \\
6 & 1.42 & 16,810 & 14.87 & 12.55 & $\checkmark$ & $\checkmark$ & 1.08 & 1.58 \\
7 & 1.24 & 5336 & 46.85 & 6.67 & $\checkmark$ & $\times$ & 1.06 & 0.96 \\
8 & 1.15 & 21,554 & 11.60 & 16.67 & $\checkmark$ & $\checkmark$ & 1.12 & 2.05 \\
LE404 & 1.03 & 31,984 & 7.83 & 6.86 & $\checkmark$ & $\times$ & 1.05 & 1.41 \\
9 & 1.47 & 14,808 & 16.88 & 1506.86 & $\checkmark$ & $\checkmark$ & 1.05 & 1.44 \\
\hline
\end{tabular}

and it is appropriate for the identification and quantification. In addition, the HPLC method can be used to verify the purity of LE404 over the duration of storage and to carry out storage stability and stress tests in the future according to ICH Guideline Topic Q 1 A (R2) [21]. These stress tests are unavoidable in the approval process for a new drug candidate. Furthermore, this method development enables a semi-preparative isolation of the degradation products and thus makes them accessible for structure analysis (e.g., NMR). After the structural analysis, the metabolites can be synthesized and are therefore accessible for essential follow-up investigations, such as toxicity studies. In addition to the liver metabolism leading to excretion, drugs can also be changed by the liver in metabolites that either have an intrinsic effect, which can differ greatly from the starting substance in terms of their effectiveness, or lead to different side effects. These can include toxic, mutagenic or carcinogenic effects [20]. For this reason, metabolites of new drug candidates must be identified and studied. The HPLC method presented here is the basis for this important research, which will take place in the future.

Funding Open Access funding enabled and organized by Projekt DEAL.

\section{Compliance with Ethical Standards}

Conflict of interest The authors declare that they have no conflicts of interest.

Human animal rights This article does not contain any studies with human participants or animals performed by any of the authors.

Open Access This article is licensed under a Creative Commons Attribution 4.0 International License, which permits use, sharing, adaptation, distribution and reproduction in any medium or format, as long as you give appropriate credit to the original author(s) and the source, provide a link to the Creative Commons licence, and indicate if changes were made. The images or other third party material in this article are included in the article's Creative Commons licence, unless indicated otherwise in a credit line to the material. If material is not included in the article's Creative Commons licence and your intended use is not permitted by statutory regulation or exceeds the permitted use, you will need to obtain permission directly from the copyright holder. To view a copy of this licence, visit http://creativecommons.org/licenses/by/4.0/.

\section{References}

1. Bonnot O, Dumas N (2014) Schizophrenic disorders in adolescence. Rev Prat 64:499-504

2. Ammon HPT, Zsilavecz MS (2014) Hunnius Vol. 11.Walter de Gruyter Verlag, Berlin

3. Schwabe U, Paffrath D (2016) Arzneiverordnungs-Report 2016. Springer-Verlag, Berlin Heidelberg

4. Lepping P, Sambhi RS, Whittington R, Lane S, Poole R (2011) Clinical relevance of findings in trials of antipsychotics: systematic review. Br J Psychiatry 198:341-345

5. Leucht S, Pitschel-Walz G, Abraham D, Kissling W (1999) Efficacy and extrapyramidal side-effects of the new antipsychotics olanzapine, quetiapine, risperidone, and sertindole compared to conventional antipsychotics and placebo. A meta-analysis of randomized controlled trials. Schizophr Res 35:51-68

6. Witt T, Hock FJ, Lehmann J (2000) 7-Methyl-6,7,8,9,14,15-hexahydro-5Hbenz[d]indolo[2,3- g]azecine: a new heterocyclic system and a new lead compound for dopamine receptor antagonists. J Med Chem 43:2079-2081

7. Mohr P, Decker M, Enzensperger C, Lehmann J (2006) Dopamine/serotonin receptor ligands. 12(1): SAR studies on hexahydro-dibenz[d, g]azecines lead to 4-chloro-7-methyl-5,6,7,8,9,14hexahydrodibenz[d, g]azecin-3-ol, the first picomolar D5-selective dopamine-receptor antagonist. J Med Chem 49:2110-2116

8. Hoefgen B, Decker M, Mohr P, Schramm AM, Rostom SA, ElSubbagh H, Schweikert PM, Rudolf DR, Kassack MU, Lehmann J (2006) Dopamine/Serotonin Receptor Ligands. 10:1 SAR studies on azecine-type dopamine receptor ligands by functional screening at human cloned D1, D2L, and D5 receptors with a microplate reader based calcium assay lead to a novel potent D1/D5 selective antagonist. J Med Chem 49:760-769

9. Schulze M, Siol O, Robaa D, Mueller FK, Enzensperger C, Fleck C, Lehmann J (2012) Molecular combination of the dopamine and serotonin scaffolds yield in novel antipsychotic drug candidatescharacterization by in vivo experiments. Drug Res 62:252-260 
10. Zergiebel S, Fleck C, Arndt HD, Enzensperger C, Seeling A (2017) Synthesis and characterization of new azecine-derivatives as potential neuroleptics. Drug Res 67:466-475

11. Zergiebel S, Arndt HD, Seeling A (2017) Optimized synthesis of new LE404- derived azecine prodrugs. Tetrahedron Lett 58:3640-3642

12. Zergiebel S, Seeling A (2018) In vitro studies on physiological and chemical stability of new LE404-derivatives with extended half-life. Drug Res 68:514-520

13. ICH Guideline (1996) I. Topic Q 2 B / Validation of analytical procedures: methodology. http://www.ich.org/products/guidelines /quality/article/quality-guidelines.html. Accessed 12 Jul 2017

14. European Pharmacopoeia 9.0 (2016) Deutscher Apotheker Verlag / Govi-Verlag-Pharmazeutischer Verlag GmbH, Eschborn

15. Lehmann J, Mohr P, Schweikert PM, Decker M, Hoefgen B/ Assignee: Friedrich- Schiller-Universität Jena, Germany. Patent: DE 102005025625 A1 2006.12.07.

16. Protokoll: thawing \& incubating human \& animal liver microsomes https://www.thermofisher.com/de/de/home/references/ protocols/drug-discovery/adme-tox-protocols/microsomes-proto col.html. Accessed 03 Apr 2018

17. Mittal B, Tulsyan S, Kumar S, Mittal RD, Agarwal G (2015) Cytochrome P450 in cancer susceptibility and treatment. Adv Clin Chem 71:77-139
18. Westerberg AW (1979) Detection and resolution of overlapped peaks for an on-line computer system for gas chromatographs. Anal Chem 41:1770-1777

19. Vemula H, Kitase Y, Ayon NJ, Bonewald L, Gutheil WG (2017) Gaussian and linear deconvolution of LC-MS/MS chromatograms of the eight aminobutyric acid isomers. Anal Biochem 516:75-85

20. Fischer D, Breitenbach J (2010) Die Pharmaindustrie EinblickDurchblick-Perspektiven, vol 3. Spektrum Akademischer Verlag, Heidelberg

21. ICH Guideline (2003) Topic Q 1 A (R2) / Stability testing of new drug substances and products; https://www.ema.europa.eu/en/ documents/scientific-guideline/ich-q-1-r2-stability-testing-newdrug-substances-products-step-5_en.pdf. Accessed 23 Nov 2020

Publisher's Note Springer Nature remains neutral with regard to jurisdictional claims in published maps and institutional affiliations. 